\title{
Systematic Review \\ Effect of Ketogenic Diet on Quality of Life in Adults with Chronic Disease: A Systematic Review of Randomized Controlled Trials
}

\author{
Myriam Abboud $^{1}$, Fatme AlAnouti ${ }^{2}$ (D) Evridiki Georgaki ${ }^{3}$ and Dimitrios Papandreou ${ }^{2, *(D)}$ \\ 1 Department of Health, College of Natural and Health Sciences, Zayed University, \\ Dubai 19282, United Arab Emirates; myriam.abboud@zu.ac.ae \\ 2 Department of Health, College of Natural and Health Sciences, Zayed University, \\ Abu Dhabi 144534, United Arab Emirates; fatme.alanouti@zu.ac.ae \\ 3 Barts and the London School of Medicine and Dentistry, Queen Mary University of London, \\ London E1 4NS, UK; evridiki.georgaki@outlook.com \\ * Correspondence: dimitrios.papandreou@zu.ac.ae
}

Citation: Abboud, M.; AlAnouti, F.; Georgaki, E.; Papandreou, D. Effect of Ketogenic Diet on Quality of Life in Adults with Chronic Disease: A Systematic Review of Randomized Controlled Trials. Nutrients 2021, 13, 4463. https://doi.org/10.3390/ nu13124463

Academic Editor: Licia Iacoviello

Received: 25 November 2021 Accepted: 10 December 2021 Published: 14 December 2021

Publisher's Note: MDPI stays neutral with regard to jurisdictional claims in published maps and institutional affiliations.

Copyright: (C) 2021 by the authors Licensee MDPI, Basel, Switzerland. This article is an open access article distributed under the terms and conditions of the Creative Commons Attribution (CC BY) license (https:// creativecommons.org/licenses/by/ $4.0 /)$.

\begin{abstract}
Background: Chronic diseases adversely affect quality of life (QOL). The ketogenic diet (KD) may improve the QOL. Objective: The aim of this systematic review was to summarize the available evidence of randomized controlled trials (RCTs) to establish the effect of KD on the QOL in adults with chronic diseases. Methods: Reporting followed PRISMA guidelines. We included randomized controlled trials (RCTs) conducted on adults with chronic disease including an intervention group that received KD and a control group, and where QOL was reported as outcome. We searched PubMed, APA PsycInfo, EMBASE, the Cumulative Index to Nursing and Allied Health Literature (CINAHL), the Cochrane Library, and Clinicaltrials.gov, and the references of the included articles and previous relevant reviews, without language or time restrictions. We critically appraised included studies and narratively synthesized their findings. Results: Nine RCTs were included. The risk of bias was low, except of allocation concealment and blinding. In patients with cancer: one RCT found an improvement in overall QOL, another reported improved physical component summary, and one found no superiority of KD in all QOL domains. In patients with neurological disorders: improved QOL was reported in Alzheimer's disease patients, whereas no difference in mental and physical health QOL was noted in patients with multiple sclerosis. In patients with obesity and type II diabetes: one RCT reported superiority of energy-restricted KD in improving role functioning, mental health, health perceptions, and pain compared with guideline-based diet, whereas in another RCT, high and low carbohydrate diets achieved comparable improvements. Among patients with knee osteoarthritis, no differences between KD and low-fat groups were noted. Dietary compliance with the KD, reported in three studies, was shown to be high. Side effects were mostly noted during the first weeks of intervention, and adverse events were not markedly different with KD and the comparison diet. Conclusions: The evidence from RCTs investigating the effect of KD on QOL in adults with chronic disease is inconclusive. The promising effect noted in some included studies and the low rates of adverse events and side effects encourage future investigations in this regard.
\end{abstract}

Keywords: diet; ketogenic; quality of life; chronic disease; systematic review; meta-analysis

\section{Introduction}

Chronic diseases are health conditions characterized by long-term physical and/or mental impairments requiring lengthy periods of supervision and care [1,2]. Professional bodies vary in the use of the term chronic, in addition to the variation in the diseases included under its umbrella [2,3]. According to the World Health Organization (WHO), a chronic disease is defined as being of long duration, slow progression, and non-transmissible [4]. 
Quality of life (QOL) is a concept approachable at varying levels of generality and the definitions are diverse [5]. It is the individuals' insights of their position in life relative to their goals and expectations [6], and its concept encompasses all domains of life including the psychological, social, and economic well-being of individuals, and their relationships to relevant features of the environment [7]. QOL is best understood as representing the gap between one's actual functional level and one's ideal standard [8]. As strongly associated with morbidity, disability, and mortality [9-11], chronic diseases affect the QOL by threatening the physical and emotional well-being, and through the development of chronic stress [12-14]. QOL is hence an important outcome in chronic health conditions [15], complimenting the traditional evaluation of reducing morbidity and mortality [16].

Although there is no standardized definition of the ketogenic diet (KD) [17], it is characterized in general by a reduction in carbohydrates $(\mathrm{CHO})$ and relative increases in the proportions of proteins and fats, enabling an increased utilization of ketones in the body $[18,19]$. The main types of the KD include the traditional ketogenic diet (TKD) containing a fixed ratio by weight of fat to combined protein and $\mathrm{CHO}$ [20], the medium-chain triglyceride (MCT) KD using MCT oil to provide around half the calories [21], and the modified Atkins diet (MAD) [22]. The clinical importance of these diets began with their successful use in the treatment of intractable childhood epilepsy [23]. Furthermore, ample evidence supports the broader therapeutic actions and effectiveness of the use of these diets in the improvement of some metabolic pathways such as cancer, type two diabetes (T2D), cardiovascular diseases (CVDs), polycystic ovary syndrome (PCOS), and some neurological disorders, leading to beneficial health effects [23-25]. However, there are still some concerns regarding their potential adverse effects including micronutrient deficiencies, appetite reduction, nausea, constipation, fatigue, hyperlipidemia, and unintended weight loss $[19,23]$. KD may improve the QOL by reducing chronic pain, inflammation, and improving metabolic parameters through multiple mechanisms [26]. The ketone bodies produced by the liver results in a greater production of ATPs with a potential increase in available energy [27], reduction in the production of reactive oxygen species [28], and inhibition of pro-inflammatory cytokine mediators [29], although conclusive evidence in this regard is lacking.

The aim of this systematic review is to summarize the available evidence of randomized controlled trials (RCTs) to establish the effect of KD on the QOL in adults with chronic diseases.

\section{Materials and Methods}

\subsection{Review Design}

This systematic review was conducted following a predefined protocol that was registered at the OSF registries (DOI: 10.17605/OSF.IO/2MK5G). The reporting of the literature searching component of the systematic review was conducted according to the Preferred Reporting Items for Systematic reviews and Meta-Analyses literature search extension (PRISMA-S) [30], and that of the systematic review according to the PRISMA statement [31]. Ethical approval for this study was not required.

\subsection{Criteria for Study Inclusion}

The inclusion criteria were designed according to the Population, Intervention, Comparator, Outcome, and Study design (PICOS) principle. Accordingly, randomized controlled trials (RCTs) thath have been conducted on adults with chronic disease; including an intervention group and received KD and a control group and assessed QOL as an outcome were included. Regarding the population, although there is a large variation in the use of the term "chronic disease" [32], studies reporting on chronic disease, defined as a disease that is long in duration, has a slow progression, and is not passed from person to person [33] were included. Studies reporting on adult patients, as defined by the investigators (e.g., aged $>18$ years at baseline) were included. Regarding the intervention, although there is no standardized definition of the KD, studies reporting on diets high 
in fat, low in $\mathrm{CHO}$ resulting in hyperketonemia [17] such as classical KD, medium-chain triglyceride (MCT)-KD, and MAD were included. When the intervention was not specified as a $\mathrm{KD}$, an upper limit of $50 \mathrm{~g}$ of $\mathrm{CHO}$ per day or $10 \%$ energy from $\mathrm{CHO}$ [34] was retained for inclusion. RCTs involving a co-intervention were included if both arms of the study received the same co-intervention. Regarding the comparator, studies employing any other type of diet (e.g., low fat diet, anti-inflammatory diet, high fiber diet, or a different form of KD) were included. Regarding the outcome, studies reporting on QOL were included irrespective of the definition adopted, nor of the assessment tool. Finally, regarding study design, only RCTs were included, irrespective of blinding.

Studies were excluded if they were conducted on healthy adults, adults with acute conditions, or on pediatric participants; were conducted on non-human species; were non-randomized or non-controlled; or were reported on in non-original articles without detailed empirical data such as posters, conference abstracts, book chapters, or reviews.

\subsection{Search Strategy}

The search strategy was validated by a medical librarian. The search involved two key concepts: (1) KD and (2) QOL. For each concept, controlled vocabulary such as Medical Subject Headings (MeSH) terms and keywords were mapped. Search terms included but were not limited to quality of life or well-being, combined with keto or Atkins. PubMed, APA PsycInfo via Ovid, EMBASE via Ovid, the Cumulative Index to Nursing and Allied Health Literature (CINAHL) via EBSCO, the Cochrane Library, and Clinicaltrials.gov were searched, without any language or publication date restriction. The literature search was conducted on 16 October 2021 by one author (MA), and the electronic search strategy run on Embase via Ovid and PubMed is available in Supplementary Material Table S1. Bibliographies of the included articles and relevant reviews were also hand-searched for eligible studies.

\subsection{Study Selection}

Two sets of authors (MA/FA; EG/DP) screened titles and/or abstracts retrieved by the search independently and in duplicate, and identified studies that potentially met the inclusion criteria. Then, the full texts of potentially eligible studies were retrieved and assessed independently and in duplicate for eligibility. Study selection was conducted following calibration exercises to ensure the validity of the process. Disagreements were solved through discussions or, in the absence of a consensus, with a third reviewer.

\subsection{Data Extraction}

The same two pairs of authors (MA/FA; EG/DP) extracted data from eligible studies, independently and in duplicate, using a data extraction form, and following a calibration exercise to ensure the validity of the process. For all included articles, the authors extracted the characteristics of the study, details of the population included (number of enrolled patients, demographic characteristics, type of chronic disease), intervention (features of KD, duration of intervention, compliance), comparator, and outcomes (QOL, adverse events, and attrition. When studies were lacking information, original references were retrieved for additional data on the design and results.

\subsection{Quality Assessment}

The same two pairs of authors (MA/FA; EG/DP) assessed the risk of bias of included studies, independently and in duplicate, following the Cochrane criteria (sequence generation, allocation concealment, blinding of participants and outcome assessors, incomplete outcome data, and selective outcome reporting) [35]. For each study, each potential source of bias was graded as low, high, or unclear risk. Disagreements were solved through consensus or with the help of a third reviewer. 


\subsection{Data Synthesis}

As a meta-analysis was not possible, the author provided a narrative synthesis of the findings from the studies including the author-recorded characteristics of the study, details of the population included, the intervention, the comparator, the assessment methods and tools as well as the study's outcomes.

\section{Results}

\subsection{Search Results}

Results of the study selection process are displayed in Figure 1. The initial search resulted in 4980 screened records, out of which nine RCTs were included in this review.

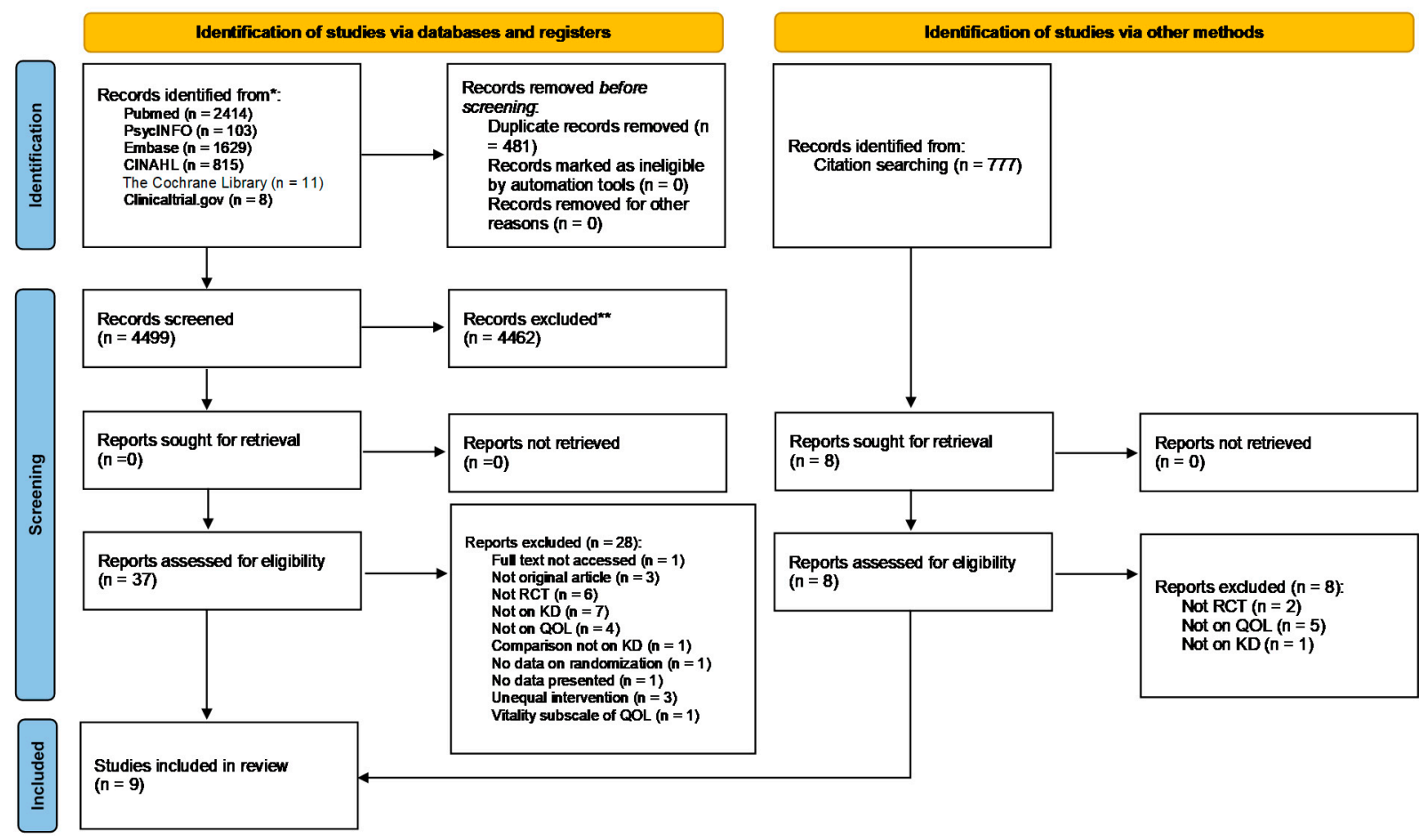

Figure 1. Preferred Reporting Items for Systematic Reviews and Meta-Analyses (PRISMA) diagram of study selection. CINAHL: Cumulative Index to Nursing and Allied Health Literature; RCT: Randomized Controlled Trial; KD: Ketogenic Diet; QOL: Quality of Life.

\subsection{Characteristics of Included Studies}

Characteristics of the included RCTs are summarized in Table 1 and detailed in Supplementary Material Table S2. Three studies were conducted in the USA [36-38], and one study took place in each of Trinidad and Tobago [36], Australia [37], Canada [38], UK [39], Iran [40], and in New Zealand [41]. Most of the studies were published within the last two years [37-39,41-44].

Regarding chronic diseases, four studies were conducted on cancer patients (several types of stages 2 and 3 cancer [36], ovarian or endometrial cancer [42], glioblastoma [39], and breast cancer [40]), two on patients with neurological disorders (multiple sclerosis [43] and Alzheimer's disease [41]), two on patients with obesity, and type II diabetes [37,38], and one on patients with knee osteoarthritis [44]. Regarding the design, only the study by Philips et al. [41], was a two-period crossover RCT.

The prescription of the KD varied between studies including a $\mathrm{CHO}$ intake less than 20 g per day [42,44], or less than 50 g per day $[37,38]$ or net $\mathrm{CHO}$ less than $6 \%$ of total energy per day [41]. Only in Durrer et al. [38] did the intervention consist of a commercial ketogenic weight loss diet plan supplemented with whole foods; whereas in Augustus et al. [36], Lee et al. [43], and Martin McGill et al. [39], the intervention con- 
sisted of MCT-KD. The duration of intervention ranged between 12 weeks [38,40-42,44] and 12 months [37]. Achievement of ketosis was measured either by blood ketones [36,37,39-44], mainly beta-hydroxybutyrate, or urinary ketones [36,39]; whereas only Strath et al. [44] did not assess ketosis. The comparators varied between standard traditional diet [36,40], energy-restricted, high $\mathrm{CHO}$ diet [37], the American Cancer Society diet [42], Diabetes Canada diet [38], modified Paleolithic diet [43], low fat diet [44], and usual diet [41,43,44]. Only Martin McGill et al. [39] compared two forms of the KD: MCT-KD and the modified MKD.

QOL was assessed using a variety of tools including disease-specific questionnaires such as the European Organization for Research and Treatment of Cancer current core questionnaire (EORTC QLQ-C30) [36,39,40], the Diabetes-39 questionnaire [37], the Multiple Sclerosis Quality of Life-54 questionnaire [43], the Quality of Life in AD questionnaire [41], and the Knee Injury and Osteoarthritis Outcome Score quality of life questionnaire [44] or generic questionnaires such as the Medical Outcomes Study Short Form (SF) [38,42].

Table 1. Characteristics of the included studies.

\begin{tabular}{|c|c|c|c|c|c|c|c|c|c|}
\hline $\begin{array}{c}\text { First Author, } \\
\text { Year, } \\
\text { Country }\end{array}$ & $\begin{array}{c}\text { Study } \\
\text { Population \& } \\
\text { Type of Chronic } \\
\text { Disease }\end{array}$ & Age; \%Male & Duration & $\begin{array}{l}\text { Intervention: } \\
\text { Features of } \\
\text { KD }\end{array}$ & Control & $\begin{array}{c}\text { Isocaloric } \\
\text { Diets (arms) }\end{array}$ & $\begin{array}{c}\text { Co- } \\
\text { intervention }\end{array}$ & $\begin{array}{l}\text { Assessment } \\
\text { of Ketosis }\end{array}$ & $\begin{array}{c}\text { Assessment } \\
\text { of QOL }\end{array}$ \\
\hline \multicolumn{10}{|c|}{ Cancer } \\
\hline $\begin{array}{c}\text { Augustus, } \\
\text { 2021, } \\
\text { Trinidad and } \\
\text { Tobago } \\
\text { (Trinidad) } \\
\text { [36] }\end{array}$ & $\begin{array}{c}\text { Stages } 2 \text { and } 3 \\
\text { cancer patients, } \\
\text { receiving } \\
\text { chemotherapy or } \\
\text { radiation, } \\
\text { nonvegetarian, } \\
\text { on a CHO-based } \\
\text { diet }(>40 \%) \\
\text { I: } n=20 ; 16 \\
\text { completers } \\
\text { C: } n=20 ; 20 \\
\text { completers }\end{array}$ & $\begin{array}{c}\text { Age: mean } \\
\text { (SD): } \\
\text { I: } \\
49.80 \pm 6.72 \\
\text { C: } \\
51.80 \pm 4.18 \\
\text { \%Male: NR }\end{array}$ & 16 weeks & $\begin{array}{l}\text { MKD: } 7 \text {-day } \\
\text { cyclic altered } \\
\text { KD }(\approx 10 \% \\
\text { CHO }(50 \mathrm{~g}), \\
15 \% \text { Protein } \\
(75 \mathrm{~g}), 75 \% \\
\text { Fat }(167 \mathrm{~g}) ; \\
2000 \mathrm{Kcal}) ; \\
\text { main source } \\
\text { of Fat: MCT }\end{array}$ & $\begin{array}{l}\text { Standard } \\
\text { traditional } \\
\text { diet }\end{array}$ & $\begin{array}{c}\text { Not } \\
\text { specified by } \\
\text { study } \\
\text { protocol }\end{array}$ & None & $\begin{array}{c}\text { Urinary } \\
\text { ketones: dip } \\
\text { stick test and } \\
\text { urine } \\
\text { analyzer }\end{array}$ & $\begin{array}{c}\text { EORTC } \\
\text { QLQ-C30 }\end{array}$ \\
\hline $\begin{array}{l}\text { Cohen, 2018, } \\
\text { Birmingham } \\
\text { (USA) [42] }\end{array}$ & $\begin{array}{c}\text { Women with } \\
\text { ovarian or } \\
\text { endometrial } \\
\text { cancer, BMI } \geq \\
18.5 \mathrm{~kg} / \mathrm{m}^{2} \\
\text { I: } n=37 ; 25 \\
\text { completers } \\
\text { C: } n=36 ; 20 \\
\text { completers }\end{array}$ & $\begin{array}{c}\text { Age: mean } \\
\text { (SD): } \\
\text { I: } 61.5 \pm 8.5 \\
\text { C: } \\
58.6 \pm 11.7 \\
\\
\text { \%Male: } 0 \%\end{array}$ & 12 weeks & 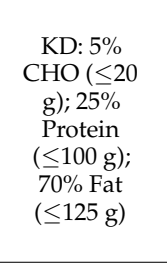 & ACS diet & $\begin{array}{l}\text { Neither } \\
\text { group was } \\
\text { instructed to } \\
\text { alter total } \\
\text { energy } \\
\text { intake }\end{array}$ & None & $\begin{array}{l}\text { Serum BHB: } \\
\text { SIRRUS } \\
\text { analyzer } \\
\text { Urinary } \\
\text { ketones: } \\
\text { strips }\end{array}$ & $\begin{array}{l}\text { SF-12 (PCS } \\
\text { and MCS) }\end{array}$ \\
\hline $\begin{array}{l}\text { Khodabakhshi, } \\
\text { 2020, Tehran } \\
\text { (Iran) [40] }\end{array}$ & $\begin{array}{l}80 \text { women with } \\
\text { locally advanced } \\
\text { or metastatic } \\
\text { breast cancer } \\
\text { receiving } \\
\text { chemotherapy } \\
\text { for } \geq 12 \text { weeks } \\
\text { I: } n=40 ; 30 \\
\text { completers } \\
\text { C: } n=40 ; 30 \\
\text { completers }\end{array}$ & $\begin{array}{c}\text { Age Range: } \\
18-70 \\
\text { I: } 44.8 \pm 8.4 \\
\text { C: } \\
45.2 \pm 15.0 \\
\\
\text { \%Male: } 0 \%\end{array}$ & 12 weeks & $\begin{array}{l}\text { 6\% CHO, } \\
\text { 19\% Protein, } \\
20 \% \text { MCT, } \\
55 \% \text { Fat }\end{array}$ & $\begin{array}{c}55 \% \mathrm{CHO}, \\
15 \% \\
\text { Protein, } \\
30 \% \text { Fat }\end{array}$ & $\begin{array}{l}\text { Both diets } \\
\text { calculated to } \\
\text { be eucaloric }\end{array}$ & None & $\begin{array}{l}\text { Blood BHB: } \\
\text { home kit }\end{array}$ & $\begin{array}{c}\text { EORTC } \\
\text { QLQ-C30 } \\
\text { and EORTC } \\
\text { QLQ-BR23 }\end{array}$ \\
\hline $\begin{array}{c}\text { Martin- } \\
\text { McGill, 2020, } \\
\text { United } \\
\text { Kingdom } \\
\text { [39] }\end{array}$ & $\begin{array}{l}12 \text { patients with } \\
\text { glioblastoma } \\
\text { planning to go } \\
\text { temozolomide } \\
\text { chemotherapy } \\
\text { and radiotherapy } \\
\text { MKD: } n=6 ; 1 \\
\text { completed } 12 \\
\text { weeks; } 1 \\
\text { completed } 12 \\
\text { months } \\
\text { MCTKD: } n=6 ; 3 \\
\text { completed } 12 \\
\text { weeks; } 2 \\
\text { completed } 12 \\
\text { months }\end{array}$ & $\begin{array}{c}\text { Age Median: } \\
\text { 57; Range: } \\
\text { 44-66 } \\
\\
\text { \%Male: } \\
66.60 \%\end{array}$ & $\begin{array}{l}12 \text { weeks } \\
12 \text { months }\end{array}$ & $\begin{array}{l}\text { I1: MKD: } 5 \% \\
\text { CHO, } 80 \% \\
\text { Fat, } 15 \% \\
\text { Protein } \\
\text { I2: MCTKD: } \\
\text { 10\% CHO, } \\
75 \% \text { Fat }(30 \% \\
\text { from MCT } \\
\text { nutrition } \\
\text { product), } \\
\text { 15\% Protein }\end{array}$ & None & $\begin{array}{c}\text { Not } \\
\text { specified by } \\
\text { study } \\
\text { protocol }\end{array}$ & None & $\begin{array}{c}\text { Urinary } \\
\text { ketones: dip } \\
\text { stick test } \\
\text { Blood } \\
\text { ketones: } \\
\text { home kit }\end{array}$ & $\begin{array}{c}\text { EORTC QLQ } \\
\text { C30 and } \\
\text { BN20 }\end{array}$ \\
\hline
\end{tabular}


Table 1. Cont.

\begin{tabular}{|c|c|c|c|c|c|c|c|c|c|}
\hline $\begin{array}{c}\text { First Author, } \\
\text { Year, } \\
\text { Country }\end{array}$ & $\begin{array}{c}\text { Study } \\
\text { Population \& } \\
\text { Type of Chronic } \\
\text { Disease }\end{array}$ & Age; \%Male & Duration & $\begin{array}{l}\text { Intervention: } \\
\text { Features of } \\
\text { KD }\end{array}$ & Control & $\begin{array}{c}\text { Isocaloric } \\
\text { Diets (arms) }\end{array}$ & $\begin{array}{c}\text { Co- } \\
\text { intervention }\end{array}$ & $\begin{array}{l}\text { Assessment } \\
\text { of Ketosis }\end{array}$ & $\begin{array}{c}\text { Assessment } \\
\text { of QOL }\end{array}$ \\
\hline \multicolumn{10}{|c|}{ Neurological disorders } \\
\hline $\begin{array}{c}\text { Lee, } 2020, \\
\text { Iowa (USA) } \\
{[43]}\end{array}$ & $\begin{array}{l}15 \text { patients with } \\
\text { relapsing } \\
\text { remitting } \\
\text { multiple sclerosis } \\
\text { or progressive } \\
\text { relapsing- } \\
\text { remitting } \\
\text { multiple sclerosis } \\
\text { (expanded } \\
\text { disability status } \\
\geq 4.5 \text { ) } \\
\text { KD: } n=5 ; 4 \\
\text { analyzed (1: } \\
\text { insufficient data) } \\
\text { MPD: } n=6 \\
\text { Usual diet: } n=4\end{array}$ & $\begin{array}{c}\text { Age } \\
\text { Total: Range: } \\
\text { 36-63 } \\
\text { Mean (SD): } \\
51.9 \pm 9.5 \\
\text { KD: } \\
51.8 \pm 11.8 \\
\text { MPD: } \\
50.3 \pm 9.5 \\
\text { C: } \\
54.5 \pm 11.8 \\
\text { \%Male: } 50 \%\end{array}$ & 12 weeks & $\begin{array}{l}\text { MCT-based } \\
\text { KD: } \\
\text { ketogenic } \\
\text { version of } \\
\text { the modified } \\
\text { Paleolithic } \\
\text { diet with } \\
\text { supplemen- } \\
\text { tal MCTs to } \\
\text { achieve a } \\
\text { daily goal of } \\
70 \% \text { of total } \\
\text { Kcal from } \\
\text { fat) }\end{array}$ & $\begin{array}{l}\text { Modified } \\
\text { Paleolithic } \\
\text { diet } \\
\text { C: Usual } \\
\text { diet }\end{array}$ & $\begin{array}{c}\text { Not } \\
\text { specified by } \\
\text { study } \\
\text { protocol }\end{array}$ & $\begin{array}{l}\text { Pre-study } \\
\text { vitamins, } \\
\text { supple- } \\
\text { ments, } \\
\text { and/or } \\
\text { medications }\end{array}$ & $\begin{array}{c}\text { Plasma BHB: } \\
\text { NR }\end{array}$ & $\begin{array}{l}\text { Multiple } \\
\text { Sclerosis } \\
\text { Quality of } \\
\text { Life-54 }\end{array}$ \\
\hline $\begin{array}{l}\text { Philips, 2021, } \\
\text { Hamilton } \\
\text { (New } \\
\text { Zealand) } \\
\text { [41] }\end{array}$ & $\begin{array}{c}26 \text { patients with } \\
\text { Alzheimer } \\
\text { diseases } \mathrm{BMI}> \\
18.5 \mathrm{~kg} / \mathrm{m}^{2} \\
\text { Phase } 1 \\
\text { KD: } n=13 ; 11 \\
\text { completers } \\
\text { Usual diet: } n=13 \text {; } \\
13 \text { completers } \\
\text { Phase } 2 \\
\text { KD: } n=13 ; 10 \\
\text { completers } \\
\text { Usual diet: } n=13 \text {; } \\
13 \text { completers }\end{array}$ & $\begin{array}{c}\text { Age } \\
\text { Total: Range: } \\
57-79 \\
\text { Mean (SD): } \\
69.8 \pm 6.0 \\
\text { KD > Usual } \\
\text { diet: Range: } \\
57-77 \\
\text { Mean(SD): } \\
68.0 \pm 5.4 \\
\text { Usual diet > } \\
\text { KD: } \\
\text { Range: } \\
61-79 \\
\text { Mean(SD): } \\
71.7 \pm 6.2 \\
\\
\% \text { Male: } \\
\text { Total: } 62 \% \\
\text { KD }>\text { Usual } \\
\text { diet: } 77 \% \\
\text { Usual diet }> \\
\text { KD: } 46 \%\end{array}$ & $\begin{array}{l}12 \text { weeks: } \\
\text { I or C } \\
10 \text { weeks: } \\
\text { washout }\end{array}$ & $\begin{array}{c}58 \% \text { Fat } \\
(26 \% \text { SFA, } \\
32 \% \text { non- } \\
\text { saturated), } \\
29 \% \text { Protein, } \\
7 \% \text { Fiber, } 6 \% \\
\text { net } \mathrm{CHO} \text { by } \\
\text { weight }\end{array}$ & $\begin{array}{c}\text { Usual diet } \\
11 \% \text { Fat } \\
\text { (3\% SFA, } \\
8 \% \text { non- } \\
\text { saturated), } \\
19 \% \\
\text { Protein, } \\
8 \% \text { Fiber, } \\
62 \% \text { net } \\
\text { CHO by } \\
\text { weight }\end{array}$ & $\begin{array}{c}\text { Not } \\
\text { specified by } \\
\text { study } \\
\text { protocol }\end{array}$ & $\begin{array}{l}\text { Daily multi- } \\
\text { vitamin }\end{array}$ & $\begin{array}{l}\text { Serum BHB: } \\
\text { ketone blood } \\
\text { monitor }\end{array}$ & QOL-AD \\
\hline \multicolumn{10}{|c|}{ Obesity and T2DM } \\
\hline $\begin{array}{c}\text { Brinkworth, } \\
\text { 2016, } \\
\text { Adelaide } \\
\text { (Australia) } \\
\text { [37] }\end{array}$ & $\begin{array}{c}\text { Adults with } \\
\text { T2DM (HbA1c } \geq \\
7.0 \% \text { or taking a } \\
\text { diabetes } \\
\text { medication), } \\
\text { overweight and } \\
\text { obese (BMI: } \\
26-45 \mathrm{~kg} / \mathrm{m}^{2} \text { ) } \\
\text { I: } n=58 ; 41 \\
\text { completers } \\
\text { C: } n=57 ; 37 \\
\text { completers }\end{array}$ & $\begin{array}{c}\text { Age Range: } \\
\text { 35-68 } \\
\text { Mean (SD) } \\
\text { I: } 58 \pm 72 \\
\text { C: } 58 \pm 7 \\
\\
\% \text { Male: } \\
\text { I: } 64 \% \\
\text { C: } 51 \%\end{array}$ & 12 months & $\begin{array}{c}\text { Very-low } \\
\text { CHO, } \\
\text { high-fat diet: } \\
14 \% \text { CHO } \\
(<50 \text { g) } ; 28 \% \\
\text { Protein, } 58 \% \\
\text { Fat }(35 \% \\
\text { MUFA, } 13 \% \\
\text { PUFA, }<10 \% \\
\text { SFA) }\end{array}$ & $\begin{array}{c}\text { High- } \\
\text { CHO, } \\
\text { low-fat } \\
\text { diet: } 53 \% \\
\text { CHO; } 17 \% \\
\text { Protein; } \\
<30 \% \text { Fat } \\
\text { (15\% } \\
\text { MUFA, } \% \\
\text { PUFA, } \\
<10 \% \text { SFA) }\end{array}$ & $\begin{array}{c}\text { For I and C: } \\
\sim 30 \% \\
\text { energy } \\
\text { restriction } \\
(500-1000 \\
\text { Kcal/day) }\end{array}$ & $\begin{array}{c}\text { 60-min, } \\
\text { moderate- } \\
\text { intensity, } \\
\text { exercise: } 3 \\
\text { days/week }\end{array}$ & $\begin{array}{c}\text { Plasma BHB: } \\
\text { D-3 Hydrox- } \\
\text { ybutyrate } \\
\text { kit }\end{array}$ & Diabetes-39 \\
\hline $\begin{array}{l}\text { Durrer, 2021, } \\
\text { Southern } \\
\text { British } \\
\text { Columbia } \\
\text { (Canada) } \\
\text { [38] }\end{array}$ & $\begin{array}{c}\text { Adults with } \\
\text { T2DM, using } \\
\text { glucose-lowering } \\
\text { medication, } \\
\text { obese }(\mathrm{BMI} \geq 30 \\
\left.\mathrm{kg} / \mathrm{m}^{2}\right) \\
\text { I: } n=98 ; 78 \\
\text { completers (98 } \\
\text { ITT) } \\
\text { C: } n=90 ; 60 \\
\text { completers ( } 90 \\
\text { ITT) }\end{array}$ & $\begin{array}{c}\text { Age: mean } \\
\text { (SD): } \\
\text { I: } 58 \pm 11 \\
\text { C: } 59 \pm 8 \\
\\
\% \text { Male: } \\
\text { I: } 44 \% \\
\text { C: } 43 \%\end{array}$ & 12 weeks & $\begin{array}{l}\text { Low-CHO } \\
\text { energy- } \\
\text { restricted } \\
\text { commercial } \\
\text { weight loss } \\
\text { plan supple- } \\
\text { mented with } \\
\text { whole foods } \\
\text { (<50 g CHO; } \\
35-45 \text { g Fat, } \\
110-120 \text { g } \\
\text { Protein; } \\
850-1100 \\
\text { Kcal) }\end{array}$ & $\begin{array}{l}\text { Information } \\
\text { conform- } \\
\text { ing with } \\
2013 \\
\text { Diabetes } \\
\text { Canada } \\
\text { Clinical } \\
\text { Practice } \\
\text { Guide- } \\
\text { lines }\end{array}$ & $\begin{array}{c}\text { Not } \\
\text { specified by } \\
\text { study } \\
\text { protocol }\end{array}$ & None & $\begin{array}{l}\text { Capillary } \\
\text { blood } \\
\text { ketones: NR }\end{array}$ & SF-20 \\
\hline
\end{tabular}


Table 1. Cont.

\begin{tabular}{|c|c|c|c|c|c|c|c|c|c|}
\hline $\begin{array}{c}\text { First Author, } \\
\text { Year, } \\
\text { Country }\end{array}$ & $\begin{array}{c}\text { Study } \\
\text { Population \& } \\
\text { Type of Chronic } \\
\text { Disease }\end{array}$ & Age; \%Male & Duration & $\begin{array}{l}\text { Intervention: } \\
\text { Features of } \\
\text { KD }\end{array}$ & Control & $\begin{array}{c}\text { Isocaloric } \\
\text { Diets (arms) }\end{array}$ & $\begin{array}{c}\text { Co- } \\
\text { intervention }\end{array}$ & $\begin{array}{c}\text { Assessment } \\
\text { of Ketosis }\end{array}$ & $\begin{array}{c}\text { Assessment } \\
\text { of QOL }\end{array}$ \\
\hline \multicolumn{10}{|c|}{ Knee Osteoarthritis } \\
\hline $\begin{array}{l}\text { Strath, 2020, } \\
\text { Birmingham } \\
\text { (USA) [44] }\end{array}$ & $\begin{array}{l}21 \text { adults with } \\
\text { knee } \\
\text { osteoarthritis } \\
\text { LCD: } n=8 \\
\text { LFD: } n=6 \\
\text { C: } n=7\end{array}$ & $\begin{array}{c}\text { Age Range: } \\
65-75 \\
\text { Mean (SD) } \\
\text { LCD: } \\
71.00 \pm 3.12 \\
\text { LFD: } \\
72.33 \pm 1.97 \\
\text { C: } \\
68.71 \pm 7.11 \\
\\
\text { \%Male: LCD: } \\
60 \% ; \text { LFD: } \\
75 \% ; \text { C: } 80 \% \\
\text { (Completers: } \\
\text { LCD: } 60 \% ; \\
\text { LFD: } 100 \% ; \\
\text { C: } 75 \%)\end{array}$ & 12 weeks & $\begin{array}{l}\text { Kcal: } \\
\text { unlimited; } \\
\text { Fat: } \\
\text { unlimited; } \\
\text { CHO: } 20 \mathrm{~g} ; \\
\text { Proteins: } \\
100 \mathrm{~g}\end{array}$ & $\begin{array}{l}\text { LFD: Kcal: } \\
\text { 800-1200; } \\
\text { Fat: 50-67 } \\
\text { g; CHO: } \\
\text { unlimited; } \\
\text { Proteins: } \\
100 \text { g } \\
\text { C: Kcal, } \\
\text { Fat, CHO, } \\
\text { Proteins: } \\
\text { unlimited }\end{array}$ & No & None & $\begin{array}{c}\text { Not } \\
\text { measured }\end{array}$ & $\begin{array}{l}\text { Knee Injury } \\
\text { and Os- } \\
\text { teoarthritis } \\
\text { Outcome } \\
\text { Score quality } \\
\text { of life }\end{array}$ \\
\hline
\end{tabular}

ACS: American Cancer Society; BHB: B-Hydroxybutyrate; BMI: Body mass index; C: Control; CHO: Carbohydrate; EORTC QLQ-C30: European Organization for Research and Treatment of Cancer current core; GHS: Global Health Status; HbA1c: Glycated hemoglobin; I: Intervention; ITT: Intention to treat; KD: Ketogenic diet; LCD: Low-carbohydrate diet; LFD: Low fat diet; MCS: Mental component summary; MCT: Medium chain triglycerides; MCTKD: Medium chain triglyceride ketogenic diet; MKD: Modified ketogenic diet; MPD: Modified Paleolithic diet; MUFA: Monounsaturated fatty acids; NR: Not reported; PCS: Physical component summary; PUFA: Polyunsaturated fatty acids; QOL: Quality of life; QOL-AD: Quality of Life in Alzheimer's Disease; SD: Standard deviation; SF-12: Medical Outcomes Study Short Form-12 Health Survey; SF-20: Medical Outcomes Study Short Form-20 Health Survey; SFA: Saturated fatty acids; T2DM: Type 2 diabetes mellitus; WHO: World Health Organization.

\subsection{Assessment of Risk of Bias}

Results of the assessment of risk of bias of the included RCTs are detailed in Figure 2. Risk of bias regarding sequence generation was low for all studies, expect for Strath et al. [44]. Allocation was concealed in Brinkworth et al. [37], Durrer et al. [38], Khodabakhshi et al. [40], Martin-McGill et al. [39], and Philips et al. [41], and was unclear in the remaining trials [36-39]. Personnel were blinded only in the studies by Brinkworth et al. [37], Lee et al. [43], and Philips et al. [41]. In general, risk of bias for blinding of outcome assessment and completeness of outcome data was low. Finally, selective reporting was suspected for the studies by Augustus et al. [36], Cohen et al. [42], Martin McGill et al. [39], and Strath et al. [44].

\subsection{Results of Included Studies}

The findings from the included studies are summarized in Table 2 and detailed in Supplementary Material Table S2. As shown in Table 2a, in patients with a diagnosis of cancer, the evidence regarding the effect of KD on QOL was inconclusive [36,39,40,42]. Augustus et al. [36] found a significant improvement in overall QOL, whereas Cohen et al. [42] reported improved QOL only at the level of physical component summary. In the study conducted by Martin McGill et al. [39], quantitative results were limited to only three patients, favoring the MKD. Finally, Khodabakhshi et al. [40] found no significant differences in the effects of KD and regular diet on all domains of QOL. 
First author, Year

\section{Augustus, 2021}

Cohen, 2018

Khodabakhshi, 2020

Martin-McGill, 2020

Lee, 2020

Philips, 2021

Brinkworth, 2016

Durrer, 2021

Strath, 2020

Low risk of bias
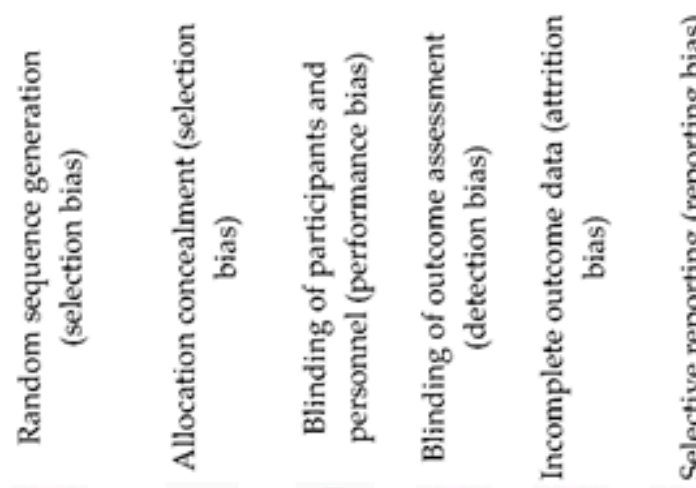

?

?
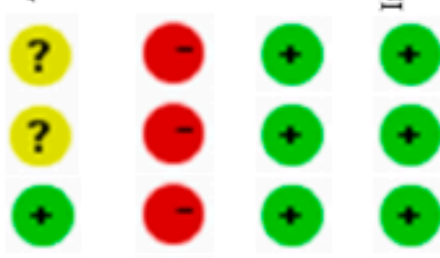

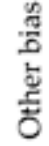
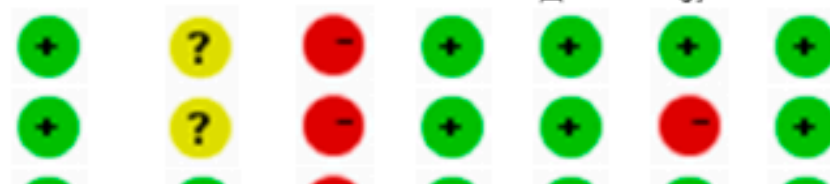

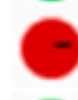
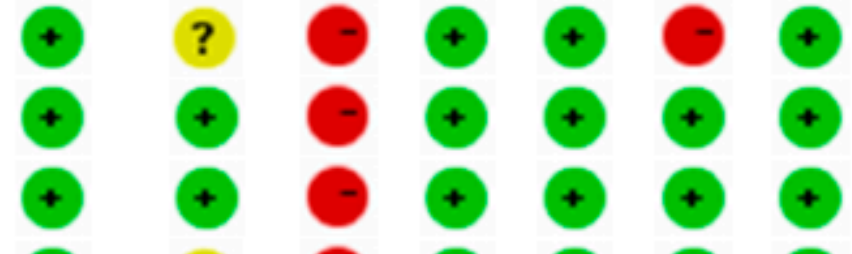

?
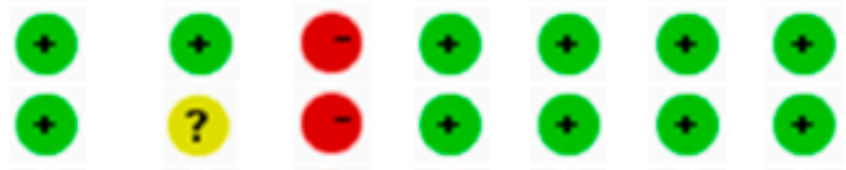

$?$
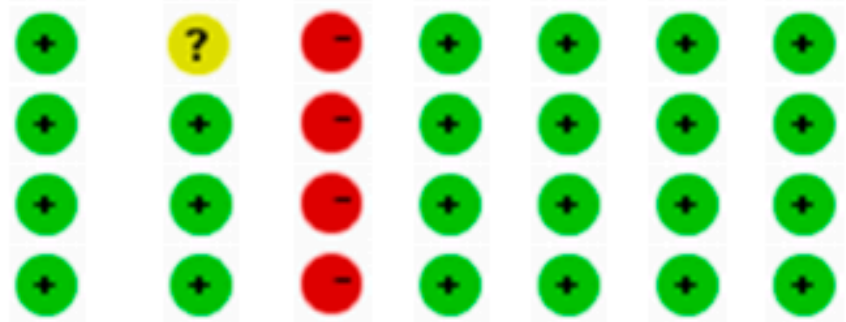

?
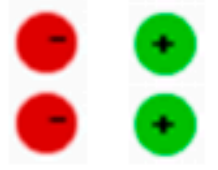

?

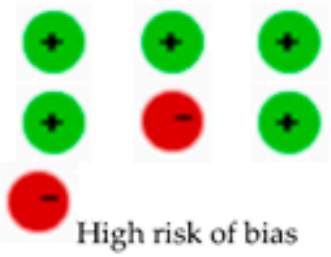

Figure 2. Risk of bias of included studies from consensus between a pair of raters [36-44].

Additionally, among patients with neurological disorders, conflicting results were noted, whereby Philips et al. [41] reported improved QOL in Alzheimer's disease patients on the KD compared with those on a usual diet, whereas Lee et al. [43] found no significant difference in mental health and physical health QOL scores among the compared groups in patients with multiple sclerosis.

Similarly, among patients with obesity and Type II diabetes [37,38], contradictory results were noted. Durrer et al. [38] showed that a ketogenic, energy-restricted diet resulted in improved measures of role functioning, mental health, health perceptions, and pain compared with a diet and lifestyle conforming with the Diabetes Clinical Practice Guidelines, whereas Brinkworth et al. [37] found that both high and low $\mathrm{CHO}$ diets achieved comparable improvements in QOL.

Finally, among patients with knee osteoarthritis [44], there were non-significant differences between the KD and low-fat groups after post hoc analysis. 
Table 2. (a) Results of included studies. (b). Results of included studies.

(a)

\begin{tabular}{|c|c|c|}
\hline First Author, Year & Effect on QOL & Conclusion \\
\hline \multicolumn{3}{|c|}{ Cancer } \\
\hline Augustus, 2021 [36] & $\begin{array}{l}\text { Mean change: I: }+28 \text { (Sig.); } \mathrm{C}:+0.6(\mathrm{NS}) \\
\text { Sig. between-group difference over time; effect } \\
\text { size: } 0.268 \text { (medium) } \\
\text { Inverse association between urinary ketones and } \\
\text { QOL }(\mathrm{b}=-3.175,95 \% \mathrm{CI}=-5.723,-0.626)\end{array}$ & $\begin{array}{l}\text { Keto-adapted patients on a MKD had an } \\
\text { improvement in self-reported QOL over time } \\
\text { KD may improve QOL of cancer patients (not } \\
\text { inclusive of advanced stage cancer) compared } \\
\text { with patients on a standard traditional diet }\end{array}$ \\
\hline
\end{tabular}

Sig. within-group increase in PCS in I $(+11 \%)$; NS

\section{change in $\mathrm{C}$}

Cohen, 2018 [42] Sig. between-group difference in adjusted PCS, NS between-group difference in MCS

NS association between PCS or MCS and serum $\mathrm{BHB}$
In women with ovarian or endometrial cancer, a KD does not negatively affect quality of life and may improve physical function

\begin{tabular}{|c|c|c|}
\hline $\begin{array}{l}\text { Khodabakhshi, } 2020 \\
\text { [40] }\end{array}$ & $\begin{array}{c}\text { Mean difference }(95 \% \mathrm{CI}) \text { : } \\
\text { Physical functioning: } 9.9(-0.7,20)(\mathrm{NS}) \\
\text { Role functioning: } 8.9(-6,23)(\mathrm{NS}) \\
\text { Cognitive functioning: } 5.5(-8,14) \\
\text { Emotional functioning: } 2(-10,14) \\
\text { Social functioning } 3.5(-4.6,5.9)(\mathrm{NS}) \\
\text { Global quality of life: } 8.1(-5.7,3.3)(\mathrm{NS})\end{array}$ & $\begin{array}{c}\text { After adjusting baseline values and chemotherapy } \\
\text { status, NS differences in all domains of QOL } \\
\text { between I and C } \\
\text { KD diet combined to chemotherapy in patients } \\
\text { with breast cancer does not bring additional } \\
\text { benefit }\end{array}$ \\
\hline $\begin{array}{l}\text { Martin-McGill, } 2020 \\
\text { [39] }\end{array}$ & $\begin{array}{l}\text { Week } 6 \text { onward, GHS improved for the patient } \\
\text { following MKD and reduced for patients } \\
\text { following MCTKD }\end{array}$ & $\begin{array}{l}\text { For retained patients at } 12 \text { months, GHS reduced } \\
\text { within the MCTKD group and improved in the } \\
\text { MKD group }\end{array}$ \\
\hline \multicolumn{3}{|c|}{ Neurological disorders } \\
\hline Lee, 2020 [43] & $\begin{array}{l}\text { NS between-group differences in mental health } \\
\text { and physical health }\end{array}$ & $\begin{array}{l}\text { NS differences in mental health and physical } \\
\text { health QOL scores among groups } \\
\text { Suggested clinically sig. improvements in mental } \\
\text { health and physical health QOL with Modified } \\
\text { Paleolithic diet (change }>5 \text { ) } \\
\text { Suggested clinically sig. decline in mental health } \\
\text { and physical health QOL with usual diet }\end{array}$ \\
\hline Philips, $2021[41]$ & 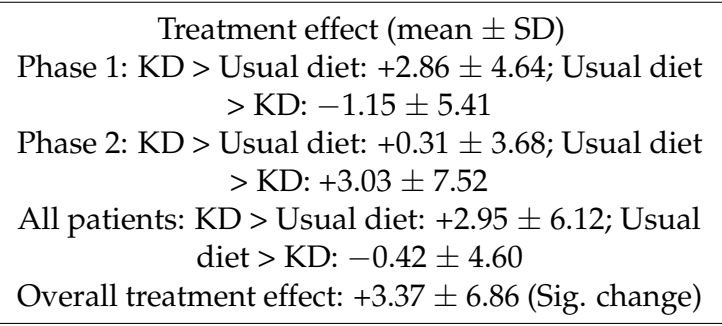 & $\begin{array}{c}\text { Patients on KD had improved QOL compared to } \\
\text { those on usual diet } \\
\text { High rates of retention and adherence are } \\
\text { achievable in applying a 12-week MKD to patients } \\
\text { with Alzheimer's disease and adverse effects are } \\
\text { mild }\end{array}$ \\
\hline \multicolumn{3}{|c|}{ Obesity and T2DM } \\
\hline Brinkworth, 2016 [37] & $\begin{array}{l}\text { NS between-group differences in anxiety and } \\
\text { worry, social burden, sexual functioning, and } \\
\text { energy and mobility }\end{array}$ & $\begin{array}{c}\text { In overweight and obese adults with T2DM, both } \\
\text { high and low CHO diets achieved comparable } \\
\text { improvements in QOL }\end{array}$ \\
\hline Durrer, $2021[38]$ & $\begin{array}{c}\text { Treatment effect }(95 \% \mathrm{CI}) \text { : } \\
\text { Physical Functioning: } 0.7(-7.7,9.9) * \\
\text { Role Functioning: } 13.6(2.4,26.3) * \\
\text { Social Functioning: } 6.1(-2,14.3) * \\
\text { Mental Health: } 6.9(1.9,12.7) * \\
\text { Health Perceptions: } 19.2(13.2,25.4)(\mathrm{NS}) \\
\text { Pain: }-7.5(-17.2,-0.1)^{*} \\
\left({ }^{*} \text { a precise } p \text {-value could not be obtained }\right)\end{array}$ & $\begin{array}{l}\text { In obese patients with T2DM, there was sig. } \\
\text { improvement in role functioning, mental health, } \\
\text { health perceptions, and pain with low-CHO } \\
\text { energy-restricted diet compared with the usual } \\
\text { diet }\end{array}$ \\
\hline
\end{tabular}


Table 2. Cont.

Knee Osteoarthritis

LCD: sig. withing-group change $(\approx-0.6)$

LFD: sig. withing-group change $(\approx-0.2)$

Strath, $2020[44]$

$\mathrm{C}$ : sig. withing-group change $(\approx-0.4)$

NS time * diet interaction and NS differences

in LCD and LFD group after post hoc analysis
NS differences in LCD and LFD group were noted after post hoc analysis

(b)

\begin{tabular}{|c|c|c|c|c|}
\hline First Author, Year & Compliance & Ketosis & $\begin{array}{c}\text { Adverse events/Side } \\
\text { effects }\end{array}$ & Attrition \\
\hline \multicolumn{5}{|c|}{ Cancer } \\
\hline Augustus, 2021 [36] & $\begin{array}{l}\text { Three-day food diaries } \\
\text { ( } 2 \text { weekdays and } 1 \\
\text { weekend) obtained at } \\
\text { the weeks } 6 \text { and } 12\end{array}$ & $\begin{array}{l}\text { Sig. rise in urinary } \\
\text { ketones in I vs. C }\end{array}$ & $\begin{array}{c}\text { I: side-effects related to } \\
\text { keto-adaptation (first } \\
\text { 2-6 weeks; sig. reduced } \\
6 \text { weeks post } \\
\text { treatment): fatigue, } \\
\text { dizziness, reduced } \\
\text { energy } \\
\text { C: } \\
\text { headaches/migraines } \\
\text { Unable to determine } \\
\text { whether reduced } \\
\text { energy or fatigue are } \\
\text { attributed to I or by } \\
\text { natural progression of } \\
\text { the disease }\end{array}$ & $\begin{array}{l}\text { I: } 2 \% \text { [ } n=4 \text { : nausea and } \\
\text { vomiting related to I affecting } \\
\text { subjects' palatability }(n=2) \text {; } \\
\text { inability to complete testing at } \\
\text { all follow-up times }(n=1) ; \\
\text { mortality not related to } \\
\text { medical treatment nor I }(n=1)] \\
\text { C: } 0 \%\end{array}$ \\
\hline
\end{tabular}

I: $n=6$ did not enroll due to scheduling conflicts; $n=6$ withdrew: 1 scheduling

Weekly phone calls/emails from the study dietitian to

Cohen, 2018 [42] review food records and discuss strategies to enhance participants' adherence
BHB (mmol/L)

I: Sig. increase

C: NS change

NR conflicts; 1 no longer wishing to comply with dietary requirements; 3 cancer recurrence; 1 death C: $n=10$ did not enroll due to scheduling conflicts; $n=6$ withdrew: 3 scheduling conflicts; 2 no longer wishing to comply with dietary requirements, 1 death

I: $n=10$ withdrew after beginning assigned diet ( 2 nausea and hypoglycemia; 3 weakness and hunger; 1 refusal to participate; 2 unable

Khodabakhshi, 2020 [40]
BHB every 3 weeks and dietary intake
Serum ketones $>0.5$

$\mathrm{mmol} / \mathrm{L}: 66.7 \%$

Sig. increase in serum ketones in I
None reported in both groups to stick to diet; 2 lack of energy and oiliness of the diet)

C: $n=3$ patients withdrew before beginning assigned diet; $n=7$ withdrew after beginning assigned diet ( 5 frequent blood sampling; 1 surgery; 1 diabetes) 
Table 2. Cont.

adherence: food diarie Assessment of ketosis:

Martin-McGill, 2020 [39]

\section{urinary ketones and}

blood ketones (at home)
Blood ketones: $\geq 4$ $\mathrm{mmol} / \mathrm{L}$

During the first 6 weeks: MCTKD: 79.7\%; MKD: $79.3 \%$
Hypokalemia $(n=2)$, hypernatremia $(n=1)$, hypocalcemia $(n=1)$, partial seizure $(n=1)$, post-operative wound infection $(n=1)$ seizure $(n=1)$, back pain $(n=1)$ [none related to the dietary intervention]

\section{Gastrointestinal side} effects:

First 6 weeks: MCT KD group: diarrhea $(n=1)$, nausea $(n=1)$, vomiting $(n=1)$, dyspepsia $(n=1)$; MKD group: vomiting $(n=1)$ and a dry mouth $(n=1)$ At month 6: MCTKD: diarrhea, dyspepsia, constipation $(n=1)$; MKD: constipation $(n=1)$
MCTKD: 6 randomized: 1 withdrew prior to commencing (changed mind); 5 commenced; 2 withdrew (1 dietary burden; 1 recruited to another trial); 3 completed 12 weeks; 1 withdrew (GI intolerance); 2 completed 12 months

MKD: 6 randomized: 1 withdrew prior to commencing (non-related SAE); 5 commenced; 4 withdrew (2 dietary burden; 1 tumor progression; 1 nausea); 1 completed 12 weeks; 1 completed 12 months

\begin{tabular}{|c|c|c|c|c|}
\hline \multicolumn{5}{|c|}{ Neurological disorders } \\
\hline Lee, 2020 [43] & Plasma BHB & $\begin{array}{c}\text { Plasma BHB: } \geq 0.50 \\
\text { mmol/L } \\
\text { Sig. higher BHB in } \\
\text { KD than MPD and } \\
\text { C }\end{array}$ & None reported & $\begin{array}{c}n=1 \text { in KD not analyzed } \\
\text { because of large amount of } \\
\text { missing data }\end{array}$ \\
\hline Philips, 2021 [41] & $\begin{array}{l}\text { Assessment of diet } \\
\text { adherence: } 3 \text {-day ( } 2 \\
\text { weekdays, } 1 \text { weekend } \\
\text { day) food record } \\
\text { Assessment of ketosis: } \\
\text { Bedtime ketone } \\
\text { monitoring }\end{array}$ & $\begin{array}{c}\text { Serum } B H B \geq 0.6 \\
\text { mmol } / \mathrm{L} \\
85.7 \% \text { of patients } \\
\text { who completed } \mathrm{KD} \\
\text { achieved sustained } \\
\text { physiological } \\
\text { ketosis }\end{array}$ & $\begin{array}{c}\text { I: Increased irritability: } \\
\text { 19\%; Increased fatigue: } \\
\text { 23\%; Sugar craving: } 8 \% \text {; } \\
\text { Insomnia: 4\%; Muscle } \\
\text { cramp: } 12 \% \text {; } \\
\text { Constipation: } 4 \% \text {; } \\
\text { Feeling light headed: } \\
\text { 15\%; Increased back } \\
\text { pain: 4\%; Excessive } \\
\text { hunger: } 8 \% \text {; Excessive } \\
\text { thirst: } 4 \% \text {; Diarrhea: } \\
\text { 4\%; Palpitations: } 4 \% \\
\text { C: Increased irritability: } \\
\text { 35\%; Increased fatigue: } \\
\text { 27\%; Sugar craving: } \\
\text { 23\%; Insomnia: } 19 \% ; \\
\text { Muscle cramp: } 4 \% ; \\
\text { Constipation: } 15 \% ; \\
\text { Feeling light headed: } \\
\text { 12\%; Increased back } \\
\text { pain: } 12 \% \text {; Nausea: } 8 \% \text {; } \\
\text { Headache: } 12 \% ; \text { Heart } \\
\text { burn: } 8 \% \text {; Palpitations: } \\
\text { 4\%; Urinary calculus: } \\
\text { 4\%; Psychotic episode: } \\
4 \%\end{array}$ & $\begin{array}{c}\text { Phase } 1 \\
\text { Pith } 1 \\
\text { I: } n=13 ; 2 \text { withdrew ( } \\
\text { declined to remove daily sugar; } \\
1 \text { excess coconut oil and } \\
\text { diarrhea); } 11 \text { completers } \\
\text { C: } n=13 ; 13 \text { completers } \\
\text { Phase } 2 \\
\text { I: } n=13 ; 10 \text { completers; } 3 \\
\text { withdrew (1 declined to } \\
\text { remove daily sugar; } 1 \text { declined } \\
\text { to remove daily beer; } 1 \\
\text { declined most of the food) } \\
\text { C: } n=13 ; 13 \text { completers }\end{array}$ \\
\hline
\end{tabular}


Table 2. Cont.

Obesity and T2DM

\begin{tabular}{|c|c|c|c|c|}
\hline $\begin{array}{c}\text { Brinkworth, } 2016 \\
\text { [37] }\end{array}$ & $\begin{array}{l}\text { Good compliance in } \\
\text { both groups to } \\
\text { prescribed diets } \\
\text { throughout the study } \\
\text { assessed by dietary } \\
\text { intake }\end{array}$ & $\begin{array}{c}\text { Plasma BHB } \\
\text { increased more } \\
\text { with I after } 4 \text { weeks } \\
\text { and remained } \\
\text { higher over } 52 \\
\text { weeks than C (Sig.) }\end{array}$ & $\begin{array}{c}\text { Musculoskeletal } \\
\text { ailments: I: } n=8 ; \text { C: } \\
n=13 \text { [Associated with } \\
\text { exercise training: I: } \\
n=6 ; \text { C: } n=8 \text { ]; } \\
\text { Gastrointestinal } \\
\text { disorders (constipation } \\
\text { and diverticulitis): I: } \\
n=2 ; \text { C: } n=1 \text {, } \\
\text { Esophageal ulcers with } \\
\text { Helicobacter pylori } \\
\text { infection: C: } n=1 ; \\
\text { Non-hospitalized } \\
\text { hypoglycemia incident: } \\
\text { I: } n=1 ; \text { Hospitalization } \\
\text { for arrhythmia with } \\
\text { suspected heart failure: } \\
\text { C: } n=1 ; \text { Prostate cancer } \\
\text { and melanoma: I: } n=1 ; \\
\text { C: } n=1 ; \text { Non-study } \\
\text { related workplace } \\
\text { injuries: I: } n=3 ; \text { C: } \\
n=1 ; \text { Hospitalization } \\
\text { for pneumonia: I: } n=1 ; \\
\text { Malignant } \\
\text { hyperthermia: I: } n=1 ; \\
\text { Anaphylactic reaction } \\
\text { to the influenza vaccine: } \\
\text { C: } n=1 ; \text { Motor vehicle } \\
\text { accident: C: } n=1\end{array}$ & $\begin{array}{c}\text { I: } n=17 \text { ( } 6 \text { lost to follow-up; } 4 \\
\text { time constraints; } 3 \text { work } \\
\text { commitments; } 2 \text { unable to } \\
\text { comply with diet; } 2 \text { personal } \\
\text { reasons; } 1 \text { health issue external } \\
\text { to study) } \\
\text { C: } n=21 \text { (4 lost to follow-up; } 1 \\
\text { time constraints; } 3 \text { work } \\
\text { commitments; } 5 \text { unable to } \\
\text { comply with diet; } 5 \text { personal } \\
\text { reasons; } 3 \text { health issue external } \\
\text { to study) }\end{array}$ \\
\hline Durrer, 2021 [38] & $\begin{array}{l}\text { I: non-adherence: } 2.12 \% \\
\text { Assessment of food } \\
\text { intake: fasting blood } \\
\text { sample and a 3-day diet }\end{array}$ & NR & $\begin{array}{c}\text { I: } n=4: \text { mild } \\
\text { hypoglycemic events } \\
\text { ( } n=2 \text { when } \\
\text { participants were } \\
\text { reluctant to reduce } \\
\text { insulin dosages by the } \\
\text { recommended amount; } \\
\text { resolved with } \\
\text { recommended } \\
\text { medication); } \\
\text { Hypoglycemic } \\
\text { symptoms ( } n=1 \text { might } \\
\text { be due to waiting too } \\
\text { long between meals; } \\
\text { resolved after solving } \\
\text { this issue); Cardiac } \\
\text { event ( } n=1 \text { occurred } 3 \\
\text { weeks into the study; } \\
\text { deemed not related to I } \\
\text { by data and safety } \\
\text { monitoring board) } \\
\text { C: } n=0\end{array}$ & $\begin{array}{c}\text { Drop-out prior to commencing } \\
\text { the trial: } \\
\text { I: } n=4 \text { ( } 1 \text { ineligible; } 3 \text { lost } \\
\text { contact }) \\
\text { C: } n=15 \text { ( } 2 \text { ineligible; } 1 \text { moved } \\
\text { away; } 12 \text { lost contact) } \\
\text { Attrition } \\
\text { I: } n=16 \text { ( } 2 \text { family issues; } 2 \\
\text { could not adhere; } 2 \text { unrelated } \\
\text { health issues; } 1 \text { travel; } 9 \text { lost } \\
\text { contact }) \\
\text { C: } n=15 \text { (15 lost contact) }\end{array}$ \\
\hline
\end{tabular}


Table 2. Cont.

Knee Osteoarthritis

\begin{tabular}{|c|c|c|c|c|}
\hline \multicolumn{5}{|c|}{ Knee Osteoarthritis } \\
\hline Strath, 2020 [44] & $\begin{array}{l}\text { Adherence verbally } \\
\text { confirmed; food } \\
\text { journals assessed by a } \\
\text { dietician and the study } \\
\text { administrator at each } \\
\text { visit }\end{array}$ & Not measured & NR & $\begin{array}{l}\text { LFD: } 1 \text { lost to nonadherence } \\
\text { C: } 2 \text { failed to complete the } \\
\text { study }\end{array}$ \\
\hline
\end{tabular}

BHB: B-Hydroxybutyrate; C: Control; CHO: Carbohydrate; CI: Confidence interval; EORTC QLQ-C30: European Organization for Research and Treatment of Cancer current core; GHS: Global Health Status; I: Intervention; KD: Ketogenic diet; LCD: Low-carbohydrate diet; LFD: Low at diet; MCS: Mental component summary; MCT: Medium chain triglycerides; MCTKD: Medium chain triglyceride ketogenic diet; MKD: Modified ketogenic diet; MPD: Modified Paleolithic diet; MUFA: Monounsaturated fatty acids; NR: Not reported; NS: Not significant; PCS: Physical component summary; PUFA: Polyunsaturated fatty acids; QOL: Quality of life; SD: Standard deviation; SFA: Saturated fatty acids; Sig: Significant; T2DM: Type 2 diabetes mellitus. * indicates interaction.

\subsection{Dietary Compliance with the Ketogenic Diet}

As Table $2 b$ shows, the results of dietary compliance with the KD were reported only in three studies $[37,38,40]$. Whenever investigated, good dietary compliance was reported [37,38,40], reaching $98 \%$ in the study by Durrer et al. [38], and $96 \%$ in the study by Khodabakhshi et al. [40]. Interestingly, in the latter study [40], only $66.7 \%$ of the group had serum ketones above $0.5 \mathrm{mmol} / \mathrm{L}$ at 12 weeks.

\subsection{Adverse Events}

Adverse events were not investigated in the studies by Cohen et al. [42] and Strath et al. [44]. In the studies conducted by Lee et al. [43] and Khodabakhshi et al. [40], no adverse events of the KD were noted. In the study by Augustus et al. [36], side-effects were mostly noted during the first two to six weeks, relating to the keto-induction phase, and they consisted mainly of feelings of fatigue and dizziness. In the studies by Brinkworth et al. [37] and Philips et al. [41], there were no marked differences in the adverse events between patients receiving KD and those receiving the comparison diet. Details about the specific side effects are reported in Table $2 b$.

\section{Discussion}

This systematic review explored the effect of KD on QOL among adults with chronic disease. Overall, we could not find conclusive evidence about the effectiveness of the different forms of the KD in improving QOL in this patient population. This finding is limited by the divergent dietary regimens used for prescribing the KD, the heterogeneity of the subjects in the studies, and chronic diseases assessed as well as the methodological variations between studies such as a comparison, and the assessment methods of ketosis and QOL.

All included publications were recent (2016-2021), reflecting increased attention to $\mathrm{KD}$ in the scientific literature for health-related outcomes beyond those related to the management of epilepsy [45]. The KD has been advocated for improving the QOL of healthy and ill people due to several biologically plausible mechanisms. One suggested theory is the effect of ketone bodies, specifically B-hydroxybutyrate, in inducing mild euphoria [46]. Qualitative findings from the study by Martin-McGill et al. [39] illustrate this, with patients reported "experiencing a fantastic quality of life" and describing the diet as offering a sense of control whilst receiving their tumor treatment". BHB has shared actions with gamma-hydroxybutyrate (GHB) on the brain. GHB is a catabolite in the brain of gamma-aminobutyrate (GABA), thus lowering cerebral energy requirements and playing a neuroprotective role [47]. Another plausible theory could be the weight-loss effect of the KD. Weight loss is suggested to improve health-related QOL; this relationship is consistent with considerable loss such as the case after bariatric surgery [48]. Furthermore, the potential positive effect of KD on QOL could be attributed to its anti-inflammatory effect. It is hypothesized that excess dietary $\mathrm{CHO}$ contribute to oxidative stress, pain, and 
inflammation [44]. Chronic inflammation significantly predicts lower QOL in emotional and relational measures $[49,50]$.

Specifically, in cancer patients, the effects of the KD on QOL could be secondary to its therapeutic effects. For example, an increasing number of preclinical studies suggest the KD as a potent anticancer therapy because of its direct effects on tumor growth, which may improve the overall health status of patients as well as their QOL [51,52]. In patients with neurological disorders such as Alzheimer's disease, although the literature is scarce and lacks scientific rigor, KD seems to improve the cognitive symptomatology of these diseases, hence its speculated effect on QOL in this patient population [53]. The possible neuroprotective effects of the KD could potentially reside in its beneficial effect on reducing accumulation of amyloid plaques, protecting against amyloid-beta toxicity, and through the modification of the neuronal network activity, although precise mechanisms remain unknown. Furthermore, BHB has cellular signaling functions [54] that broadly link the outside environment to cellular function through epigenetic gene regulation, with potential implications on human aging [55]. Finally, the KD diet is reportedly associated with improvements in food cravings, increased levels of physical activity, sleep, and sexual function, and decreased medication need $[45,56]$. These effects may be of utmost interest to type 2 diabetic patients.

The scientific literature highlights the lack of a standardized definition of the KD. Commonly, this diet is characterized by a reduction in $\mathrm{CHO}$ below $10 \%$ of daily energy intake or typically 30 to $50 \mathrm{~g}$ of $\mathrm{CHO}$ per day, and a relative increase in fat intake, with a fat-to-CHO and protein intake ratio of 3:1 to 4:1 [57]. The included studies in this systematic review varied in their definition of the $\mathrm{KD}$, the level of $\mathrm{CHO}$ restriction as well as protein and fat intakes, in addition to the inclusion of MCT as a source of fat. Furthermore, the use of KD and induction of ketosis were not explicit in some included studies, nevertheless, the features of the interventional diet were in accordance with that of a KD.

There was also a variation in the definition of physiological ketosis with different cut-off values used in different studies as well as the assessment of ketosis, varying between blood and urinary ketones. Specifically, regarding the latter method, urinary ketones were used to monitor the diet in numerous studies $[36,39,42]$. Urinary ketones may not be robust markers of compliance, as they can be affected by hydration status, some medications or substances such as vitamin C. Furthermore, the reading is an average of urine ketone levels since the last void. Finally, some kits only measure acetoacetic levels and others have a short shelf-life [56]. Of interest, apparently low urinary ketones may discourage patients when they are adhering to the diet robustly [39]; this is to be considered for future RCTs investigating the KD. In addition, only the two studies conducted by Augustus et al. [36] and Cohen et al. [42] reported on the relationship between ketone bodies and QOL. The results of the two studies were contradictory: while the first reported an inverse association between urinary ketones and QOL, the latter reported no association between physical nor mental health and serum BHB, hence a conclusion on this relationship cannot be drawn.

Importantly, participant blinding was impossible in all trials. It is thus likely that improvements noted with the KD may be biased by the increased attention given to patients through consultations, education, and follow-up, and by the fact that patients had the sense that they were actively participating in treatment to enhance their disease condition [36]. These factors were not measured in the trials, hence cannot be controlled with the analysis. Furthermore, remarkable adherence was noted in this systematic review. This finding should be interpreted with caution since only three studies (i.e., Brinkworth et al. [37], Durrer et al. [38], and Khodabakhshi et al. [40]) reported the results of the dietary compliance, and in these studies, compliance was self-reported, which may be subject to recall bias, error and inaccuracy, or social desirability bias [58]. Low adherence to the KD in adult patients with cancer was reported in a recent systematic review (49\%) [25]. Adherence to the KD requires drastic changes, which could hinder long-term compliance [59]. Indeed, Augustus et al. [36] presented the case that the KD may not be offered to all patients and 
that being unable to adhere to the KD or struggling to be adapted to it may adversely affect the QOL and mental health of patients.

Interestingly, in the majority of included studies, adverse events did not greatly differ between the KD and its comparison. Furthermore, commonly reported side effects with the KD included fatigue, dizziness, and gastrointestinal symptoms. These effects, mainly reported in the first weeks of adopting the $\mathrm{KD}$, are attributed to the keto-induction period, which varies greatly inter-individual [60,61]. Specifically, Augustus et al. [36] reported a small but distinct gender-based difference in this period, whereby men adapted faster to the KD as well as an age-based difference, with younger individuals adapting faster than their older counterparts. The keto-induction period involves a course of immediate side-effects known as the keto-flu, which coincide with a high level of ketone bodies. These effects steadily attenuate over time with keto-adaptation (i.e., organ homeostasis to using ketones as the primary source of energy [60]).

\section{Strengths and Limitations}

This was the first study to compile evidence from RCTs on the effect of KD diet on QOL in adults with chronic diseases. We adopted a sensitive search strategy, followed the recommended methods for applying the search, selection of studies, data extraction, and quality assessment as well as best-practices for the reporting of the review [30,31]. Moreover, when studies were lacking information, original references were retrieved for additional data on design and results. Finally, the risk of bias of the majority of included studies was low, except for the allocation concealment and blinding of participants and personnel.

On the other hand, the review is limited by the heterogeneity of included studies, which complicates the comparisons and the interpretation of the findings, specifically regarding assessed populations, assessment methods, and study outcomes. Additionally, some studies lacked information crucial for the interpretation of the results such as baseline QOL values, adverse events, assessment of ketosis, and proportion of participants with sustained physiological ketosis throughout the trial. Having such information would have helped us interpret the findings in a better manner. Moreover, the search, despite being highly broad and sensitive, might have missed some relevant studies. This limitation is common to systematic reviews. Other limitations related to being unable to access one potentially eligible study for full text screening, and excluding additional two studies in this phase due to lack of information on randomization and unavailability of outcome data in the published article. We tried to contact the respective authors, without an answer. Finally, given the heterogeneity of the studies included, meta-analyzing their results was impossible.

\section{Conclusions}

In conclusion, the evidence from RCTs investigating the effect of KD on QOL in adults with chronic disease is inconclusive. The promising effect noted in some included studies, and the low rates of adverse events and side effects encourage future investigations in this regard. Hence, additional high-quality, powered trials with long enough follow-up periods, are warranted to elucidate the effect of the KD on QOL in adults with chronic disease and explore the optimal diet composition and timing of initiation for optimal outcomes.

Supplementary Materials: The following are available online at https:/ / www.mdpi.com/article/10 $.3390 /$ nu13124463/s1, Table S1: Characteristics of included studies., Table S2: a. Results of included studies; b. Results of included studies.

Author Contributions: Conceptualization, M.A. and D.P.; running the search, M.A.; study selection, data extraction, quality assessment, M.A., F.A., E.G. and D.P.; writing-original draft preparation, M.A.; writing-review and editing F.A., E.G. and D.P.; funding acquisition, M.A., F.A. and D.P. All authors have read and agreed to the published version of the manuscript. 
Funding: College of Natural and Health Sciences, Zayed University, Dubai, United Arab Emirates. The funding body will not be involved in the design of the study and collection, analysis, and interpretation of data or in writing the manuscript.

Institutional Review Board Statement: Not applicable.

Informed Consent Statement: Not applicable.

Conflicts of Interest: The authors declare no conflict of interest.

\section{References}

1. Reynolds, R.; Dennis, S.; Hasan, I.; Slewa, J.; Chen, W.; Tian, D.; Bobba, S.; Zwar, N. A systematic review of chronic disease management interventions in primary care. BMC Fam. Pract. 2018, 19, 11. [CrossRef]

2. Schmidt, H. Chronic Disease Prevention and Health Promotion. In Public Health Ethics: Cases Spanning the Globe; Dawson, A., Saenz, C., Reis, A., Bolan, G., Eds.; Springer International Publishing: Cham, Switzerland, 2016.

3. Cugusi, L.; Prosperini, L.; Mura, G. Exergaming for Quality of Life in Persons Living with Chronic Diseases: A Systematic Review and Meta-analysis. PMER 2021, 13, 756-780.

4. World Health Organization. Global Status Report on Noncommunicable Diseases 2014; World Health Organization: Geneva, Switzerland, 2014.

5. Felce, D.; Perry, J. Quality of life: Its definition and measurement. Res. Dev. Disabil. 1995, 16, 51-74. [CrossRef]

6. World Health Organization. Programme on Mental Health: WHOQOL User Manual; World Health Organization: Geneva, Switzerland, 1998.

7. Fallowfield, L. What is Quality of Life. Available online: http://www.bandolier.org.uk/painres/download/What $\% 20$ is $\% 202009$ /What_is_QOL.pdf (accessed on 6 October 2021).

8. Cella, D.F. Quality of life: Concepts and definition. J. Pain Symptom Manag. 1994, 9, 186-192. [CrossRef]

9. Walker, A.E. Multiple chronic diseases and quality of life: Patterns emerging from a large national sample, Australia. Chronic Illn. 2007, 3, 202-218. [CrossRef] [PubMed]

10. Murray, C.J.; Lopez, A.D.; World Health Organization. The Global Burden of Disease: A Comprehensive Assessment of Mortality and Disability from Diseases, Injuries, and Risk Factors in 1990 and Projected to 2020: Summary; World Health Organization: Geneva, Switzerland, 1996.

11. Yach, D.; Hawkes, C.; Gould, C.L.; Hofman, K.J. The global burden of chronic diseases: Overcoming impediments to prevention and control. JAMA 2004, 291, 2616-2622. [CrossRef]

12. Glozman, J.M. Quality of life of caregivers. Neuropsychol. Rev. 2004, 14, 183-196. [CrossRef]

13. Berry, R.A.; Murphy, J.F. Well-being of caregivers of spouses with Parkinson's disease. Clin. Nurs. Res. 1995, 4, 373-386. [CrossRef] [PubMed]

14. Carter, J.H.; Stewart, B.J.; Archbold, P.G.; Inoue, I.; Jaglin, J.; Lannon, M.; Rost-Ruffner, E.; Tennis, M.; McDermott, M.P.; Amyot, D.; et al. Living with a person who has Parkinson's disease: The spouse's perspective by stage of disease. Parkinson's Study Group. Mov. Disord. Off. J. Mov. Disord. Soc. 1998, 13, 20-28. [CrossRef]

15. Bruce, S.; Devlin, A.; Air, L.; Cook, L. Changes in quality of life as a result of ketogenic diet therapy: A new approach to assessment with the potential for positive therapeutic effects. Epilepsy Behav. 2017, 66, 100-104. [CrossRef] [PubMed]

16. Brundage, M.; Leis, A.; Bezjak, A.; Feldman-Stewart, D.; Degner, L.; Velji, K.; Zetes-Zanatta, L.; Tu, D.; Ritvo, P.; Pater, J. Cancer patients' preferences for communicating clinical trial quality of life information: A qualitative study. Qual. Life Res. Int. J. Qual. Life Asp. Treat. Care Rehabil. 2003, 12, 395-404. [CrossRef] [PubMed]

17. Bouteldja, N.; Andersen, L.T.; Møller, N.; Gormsen, L.C. Using positron emission tomography to study human ketone body metabolism: A review. Metab. Clin. Exp. 2014, 63, 1375-1384. [CrossRef] [PubMed]

18. Paoli, A.; Rubini, A.; Volek, J.S.; Grimaldi, K.A. Beyond weight loss: A review of the therapeutic uses of very-low-carbohydrate (ketogenic) diets. Eur. J. Clin. Nutr. 2013, 67, 789-796. [CrossRef] [PubMed]

19. Veech, R.L. The therapeutic implications of ketone bodies: The effects of ketone bodies in pathological conditions: Ketosis, ketogenic diet, redox states, insulin resistance, and mitochondrial metabolism. Prostaglandins Leukot. Essent. Fat. Acids 2004, 70, 309-319. [CrossRef]

20. Dhamija, R.; Eckert, S.; Wirrell, E. Ketogenic diet. Can. J. Neurol. Sci. 2013, 40, 158-167. [CrossRef]

21. Neal, E.G.; Chaffe, H.; Schwartz, R.H.; Lawson, M.S.; Edwards, N.; Fitzsimmons, G.; Whitney, A.; Cross, J.H. The ketogenic diet for the treatment of childhood epilepsy: A randomised controlled trial. Lancet Neurol. 2008, 7, 500-506. [CrossRef]

22. Coppola, G.; D'Aniello, A.; Messana, T.; Di Pasquale, F.; della Corte, R.; Pascotto, A.; Verrotti, A. Low glycemic index diet in children and young adults with refractory epilepsy: First Italian experience. Seizure 2011, 20, 526-528. [CrossRef] [PubMed]

23. Kessler, S.K.; Neal, E.G.; Camfield, C.S.; Kossoff, E.H. Dietary therapies for epilepsy: Future research. Epilepsy Behav. 2011, 22, 17-22. [CrossRef]

24. Oliveira, C.L.P.; Mattingly, S.; Schirrmacher, R.; Sawyer, M.B.; Fine, E.J.; Prado, C.M. A Nutritional Perspective of Ketogenic Diet in Cancer: A Narrative Review. J. Acad. Nutr. Diet. 2018, 118, 668-688. [CrossRef] 
25. Sremanakova, J.; Sowerbutts, A.M.; Burden, S. A systematic review of the use of ketogenic diets in adult patients with cancer. J. Hum. Nutr. Diet. Off. J. Br. Diet. Assoc. 2018, 31, 793-802. [CrossRef]

26. Field, R.; Pourkazemi, F.; Rooney, K. Effects of a low-carbohydrate ketogenic diet on reported pain, blood biomarkers and quality of life in patients with chronic pain: A pilot randomised clinical trial rationale, study design and protocol. Eur. J. Integr. Med. 2021, 45, 101346. [CrossRef]

27. Rho, J.M. How does the ketogenic diet induce anti-seizure effects? Neurosci. Lett. 2017, 637, 4-10. [CrossRef] [PubMed]

28. Miller, V.J.; Villamena, F.A.; Volek, J.S. Nutritional Ketosis and Mitohormesis: Potential Implications for Mitochondrial Function and Human Health. J. Nutr. Metab. 2018, 2018, 5157645. [CrossRef]

29. Masino, S.A.; Ruskin, D.N. Ketogenic diets and pain. J. Child Neurol. 2013, 28, 993-1001. [CrossRef]

30. Rethlefsen, M.L.; Kirtley, S.; Waffenschmidt, S.; Ayala, A.P.; Moher, D.; Page, M.J.; Koffel, J.B. PRISMA-S: An extension to the PRISMA Statement for Reporting Literature Searches in Systematic Reviews. Syst. Rev. 2021, 10, 39. [CrossRef] [PubMed]

31. Page, M.J.; McKenzie, J.E.; Bossuyt, P.M.; Boutron, I.; Hoffmann, T.C.; Mulrow, C.D.; Shamseer, L.; Tetzlaff, J.M.; Akl, E.A.; Brennan, S.E.; et al. The PRISMA 2020 statement: An updated guideline for reporting systematic reviews. J. Clin. Epidemiol. 2021, 134, 178-189. [CrossRef]

32. Bernell, S.; Howard, S.W. Use Your Words Carefully: What Is a Chronic Disease? Front. Public Health 2016, 4, 159. [CrossRef]

33. GACD. Non-Communicable Diseases: Key Facts and Figures. Available online: https://www.gacd.org/about/what-we-do/ what-are-ncds/key-facts-and-figures (accessed on 6 October 2021).

34. Crosby, L.; Davis, B.; Joshi, S.; Jardine, M.; Paul, J.; Neola, M.; Barnard, N.D. Ketogenic Diets and Chronic Disease: Weighing the Benefits Against the Risks. Front. Nutr. 2021, 8, 702802. [CrossRef]

35. Higgins, J.P.; Thomas, J.; Chandler, J.; Cumpston, M.; Li, T.; Page, M.J.; Welch, V.A. Cochrane Handbook for Systematic Reviews of Interventions; John Wiley \& Sons: Chichester, UK, 2019.

36. Augustus, E.; Granderson, I.; Rocke, K.D. The Impact of a Ketogenic Dietary Intervention on the Quality of Life of Stage II and III Cancer Patients: A Randomized Controlled Trial in the Caribbean. Nutr. Cancer 2021, 73, 1590-1600. [CrossRef] [PubMed]

37. Brinkworth, G.D.; Luscombe-Marsh, N.D.; Thompson, C.H.; Noakes, M.; Buckley, J.D.; Wittert, G.; Wilson, C.J. Long-term effects of very low-carbohydrate and high-carbohydrate weight-loss diets on psychological health in obese adults with type 2 diabetes: Randomized controlled trial. J. Intern. Med. 2016, 280, 388-397. [CrossRef]

38. Durrer, C.; McKelvey, S.; Singer, J.; Batterham, A.M.; Johnson, J.D.; Gudmundson, K.; Wortman, J.; Little, J.P. A randomized controlled trial of pharmacist-led therapeutic carbohydrate and energy restriction in type 2 diabetes. Nat. Commun. 2021, $12,5367$. [CrossRef] [PubMed]

39. Martin-McGill, K.J.; Marson, A.G.; Tudur Smith, C.; Young, B.; Mills, S.J.; Cherry, M.G.; Jenkinson, M.D. Ketogenic diets as an adjuvant therapy for glioblastoma (KEATING): A randomized, mixed methods, feasibility study. J. Neuro-Oncol. 2020, 147, 213-227. [CrossRef] [PubMed]

40. Khodabakhshi, A.; Seyfried, T.N.; Kalamian, M.; Beheshti, M.; Davoodi, S.H. Does a ketogenic diet have beneficial effects on quality of life, physical activity or biomarkers in patients with breast cancer: A randomized controlled clinical trial. Nutr. J. 2020, 19, 87. [CrossRef] [PubMed]

41. Phillips, M.C.L.; Deprez, L.M.; Mortimer, G.M.N.; Murtagh, D.K.J.; McCoy, S.; Mylchreest, R.; Gilbertson, L.J.; Clark, K.M.; Simpson, P.V.; McManus, E.J.; et al. Randomized crossover trial of a modified ketogenic diet in Alzheimer's disease. Alzheimer's Res. Ther. 2021, 13, 51. [CrossRef]

42. Cohen, C.W.; Fontaine, K.R.; Arend, R.C.; Soleymani, T.; Gower, B.A. Favorable Effects of a Ketogenic Diet on Physical Function, Perceived Energy, and Food Cravings in Women with Ovarian or Endometrial Cancer: A Randomized, Controlled Trial. Nutrients 2018, 10, 1187. [CrossRef]

43. Lee, J.E.; Titcomb, T.J.; Bisht, B.; Rubenstein, L.M.; Louison, R.; Wahls, T.L. A Modified MCT-Based Ketogenic Diet Increases Plasma $\beta$-Hydroxybutyrate but Has Less Effect on Fatigue and Quality of Life in People with Multiple Sclerosis Compared to a Modified Paleolithic Diet: A Waitlist-Controlled, Randomized Pilot Study. J. Am. Coll. Nutr. 2021, 40, 13-25. [CrossRef] [PubMed]

44. Strath, L.J.; Jones, C.D.; Philip George, A.; Lukens, S.L.; Morrison, S.A.; Soleymani, T.; Locher, J.L.; Gower, B.A.; Sorge, R.E. The Effect of Low-Carbohydrate and Low-Fat Diets on Pain in Individuals with Knee Osteoarthritis. Pain Med. 2020, 21, 150-160. [CrossRef]

45. McDonald, T.J.W.; Cervenka, M.C. Lessons learned from recent clinical trials of ketogenic diet therapies in adults. Curr. Opin. Clin. Nutr. Metab. Care 2019, 22, 418-424. [CrossRef]

46. Brown, A.J. Low-carb diets, fasting and euphoria: Is there a link between ketosis and gamma-hydroxybutyrate (GHB)? Med. Hypotheses 2007, 68, 268-271. [CrossRef]

47. Cash, C.D. Gamma-hydroxybutyrate: An overview of the pros and cons for it being a neurotransmitter and/or a useful therapeutic agent. Neurosci. Biobehav. Rev. 1994, 18, 291-304. [CrossRef]

48. Kolotkin, R.L.; Andersen, J.R. A systematic review of reviews: Exploring the relationship between obesity, weight loss and health-related quality of life. Clin. Obes. 2017, 7, 273-289. [CrossRef] [PubMed]

49. de Almeida Roediger, M.; de Fátima Nunes Marucci, M.; Duim, E.L.; Santos, J.L.F.; de Oliveira Duarte, Y.A.; de Oliveira, C. Inflammation and quality of life in later life: Findings from the health, well-being and aging study (SABE). Health Qual. Life Outcomes 2019, 17, 26. [CrossRef] [PubMed] 
50. Nowakowski, A.C. Chronic inflammation and quality of life in older adults: A cross-sectional study using biomarkers to predict emotional and relational outcomes. Health Qual. Life Outcomes 2014, 12, 141. [CrossRef] [PubMed]

51. Shingler, E.; Perry, R.; Mitchell, A.; England, C.; Perks, C.; Herbert, G.; Ness, A.; Atkinson, C. Dietary restriction during the treatment of cancer: Results of a systematic scoping review. BMC Cancer 2019, 19, 811. [CrossRef] [PubMed]

52. Weber, D.D.; Aminzadeh-Gohari, S.; Tulipan, J.; Catalano, L.; Feichtinger, R.G.; Kofler, B. Ketogenic diet in the treatment of cancer-Where do we stand? Mol. Metab. 2020, 33, 102-121. [CrossRef] [PubMed]

53. Pavón, S.; Lázaro, E.; Martínez, O.; Amayra, I.; López-Paz, J.F.; Caballero, P.; Al-Rashaida, M.; Luna, P.M.; García, M.; Pérez, M.; et al. Ketogenic diet and cognition in neurological diseases: A systematic review. Nutr. Rev. 2021, 79, 802-813. [CrossRef] [PubMed]

54. Newman, J.C.; Verdin, E. $\beta$-Hydroxybutyrate: A Signaling Metabolite. Annu. Rev. Nutr. 2017, 37, 51-76. [CrossRef]

55. Martin, C.; Preedy, V.; Rajendram, R. (Eds.) Factors Affecting Neurological Aging; Academic Press: Cambridge, MA, USA, 2021.

56. Dhatariya, K. Blood Ketones: Measurement, Interpretation, Limitations, and Utility in the Management of Diabetic Ketoacidosis. Rev. Diabet. Stud. RDS 2016, 13, 217-225. [CrossRef]

57. Castro-Barquero, S.; Ruiz-León, A.M.; Sierra-Pérez, M.; Estruch, R.; Casas, R. Dietary Strategies for Metabolic Syndrome: A Comprehensive Review. Nutrients 2020, 12, 2983. [CrossRef]

58. Nieman, D. Nutritional Assessment, 7th ed.; McGraw-Hill: New York, NY, USA, 2019.

59. Grammatikopoulou, M.G.; Goulis, D.G.; Gkiouras, K.; Theodoridis, X.; Gkouskou, K.K.; Evangeliou, A.; Dardiotis, E.; Bogdanos, D.P. To Keto or Not to Keto? A Systematic Review of Randomized Controlled Trials Assessing the Effects of Ketogenic Therapy on Alzheimer Disease. Adv. Nutr. 2020, 11, 1583-1602. [CrossRef]

60. Bostock, E.C.S.; Kirkby, K.C.; Taylor, B.V.; Hawrelak, J.A. Consumer Reports of "Keto Flu" Associated With the Ketogenic Diet. Front. Nutr. 2020, 7, 20. [CrossRef] [PubMed]

61. Batch, J.T.; Lamsal, S.P.; Adkins, M.; Sultan, S.; Ramirez, M.N. Advantages and Disadvantages of the Ketogenic Diet: A Review Article. Cureus 2020, 12, e9639. [CrossRef] [PubMed] 